\title{
¿Perfiles profesionales 2.0? Una aproximación a la correlación entre la demanda laboral y la formación universitaria
}

\author{
Hada M. SÁnChez GonZALES \\ Universidad de Sevilla \\ misago@us.es \\ Sandra MÉNDEZ MuROS \\ Universidad de Sevilla \\ sanmenmur@us.es
}

Recibido: 04/10/2012

Aceptado: 23/01/2013

Resumen

El desarrollo de la Web 2.0 y de los medios sociales ha provocado el cambio del paradigma comunicativo y ha permitido que las empresas mantengan una relación directa con el usuario. El presente artículo analiza la oferta educativa que ofrecen los másteres oficiales de las universidades españolas y su conexión con las demandas laborales. A través del uso de técnicas cualitativas y cuantitativas, que permiten obtener validez y fiabilidad mediante la triangulación metodológica, se intenta responder a cuestiones como cuáles son los nuevos perfiles profesionales bajo demanda y si las universidades españolas han asumido el cambio generado en Internet. Se concluye que no es concurrente la demanda laboral de los nuevos perfiles gestados en la Red con la oferta educativa universitaria y más aún por parte de las instituciones públicas.

Palabras clave: Web social, demanda laboral, universidad, periodismo, perfiles profesionales.

\section{Professional Profiles 2.0? An Approach to the Correlation between the Labour Demand and the University Education}

\begin{abstract}
The development the Web 2.0 and the social media have given rise to the communicative paradigm change and it has made possible to the companies support a direct relation with the user. The current paper analyses the educational supply that the Official Masters of the Spanish Universities and the link with labour demands. By means of the use of qualitative and quantitative techniques, that allow us to reach a very high reliability applying the methodological triangulation, we try to answer questions as which are the new professional profiles under demand and if Spanish Universities have the assumed the change generated on Internet. It has been concluded that the labour demand of the new profiles created on the Net is not concurrent with the university offer and even more from the public institutions.

Keywords: Social Web, Labour Demand, University, Journalism, Professional Profiles.

\section{Referencia normalizada}

SÁNCHEZ GONZALES, Hada M. y MÉNDEZ MUROS, Sandra (2013): “¿Perfiles profesionales 2.0? Una aproximación a la correlación entre la demanda laboral y la formación universitaria". Estudios sobre el mensaje periodístico. Vol. 19. Núm. especial abril, págs.: 981-993. Madrid, Servicio de Publicaciones de la Universidad Complutense.
\end{abstract}

Sumario: 1. Introducción. 2. Material y métodos. 3. Perfiles profesionales bajo demanda. 4. Discusión. 5. Referencias bibliográficas. 


\section{Introducción}

Internet cobra cada día mayor protagonismo en todos los ámbitos de la vida de las personas, es decir, en el plano social, profesional, educativo, político, económico, etc. El imparable desarrollo de la Web 2.0 y la eclosión de los "social media" han cambiado los parámetros tradicionales de transmitir el mensaje. La comunicación multidireccional y multimedia, la convergencia e instantaneidad, la ubicuidad y la inteligencia colectiva y emocional son algunas de las características que se pueden percibir dentro de este espacio donde no existe frontera o barrera que impida una efectiva comunicación.

La Web social, como generador de la democratización digital, ha cambiado el hábito de las personas hacia nuevas formas de relación, información y comunicación, tal es así que el número de internautas ha aumentado un 7,1 por ciento en el último año y se acerca a los 22,2 millones de personas. Resultado que podría derivarse del porcentaje de hogares españoles con acceso a Internet, que asciende al 59,1 por ciento frente al 54,0 por ciento del año anterior, según el estudio del Instituto Nacional de Estadística (2011: web) sobre Equipamiento y Uso de Tecnologías de Información y Comunicación en los Hogares de 2010.

Los medios sociales, potenciados por la innovación tecnológica, se constituyen en el fenómeno emergente sociocultural de esta época porque han cambiado el hábito de consumo de los internautas provocando que se origine una sociedad hiperconectada. Sin embargo, habría que aclarar que el perfil de los usuarios que más tiempo pasan en lnternet poseen las edades comprendidas entre 25 y 34 años con un 26,1 por ciento y entre 35 - 44 años con el 23,6 por ciento por encima del resto, según el Estudio General de Medios (EGM) (AIMC, 2011: web).

La sociedad en Red avanza hacia audiencias cada vez más activas inmersas no sólo en el proceso de participación, sino en la producción y distribución de los mensajes. Los medios sociales son los artífices del cambio que han protagonizado los usuarios dentro del proceso de comunicación, es decir, dejan de ser receptores pasivos para convertirse en agentes productores del mensaje, de tal forma que el 70 por ciento de los internautas en España se ha registrado en alguna red social, a diferencia del año anterior que no llegó al 51 por ciento. Facebook se sitúa en primer lugar con el 89 por ciento entre los encuestados que utiliza o visita la red social; le sigue Youtube con el 60 por ciento y, en tercer lugar, se encuentra Tuenti con el 44 por ciento. El "microbloggin" Twitter ha logrado un incremento mejorando los resultados del año pasado hasta alcanzar el 50 por ciento actual. Así lo señala el "II Estudio sobre Redes Sociales en Internet", realizado por Interactive Advertising Bureau (IAB-Elogia Ipsofacto, 2010: web).

A la vista de los datos, es necesario explicar qué son los medios sociales, qué expresa el término en cuestión. Hablamos de un cambio de paradigma que no tiene nada que ver con el término "mass media" que se ha venido utilizando durante algunos años. Se trata de medios de comunicación social o conjunto de plataformas digitales y sociales que son generados por los usuarios a través de la publicación e intercambio de información multimedia. Son espacios que permiten la comunicación instantánea y la inteligencia emocional. 
Es una tendencia comunicativa que ha despertado el interés de empresas, instituciones y medios de comunicación porque les permite establecer nuevos vínculos con el usuario según los intereses y necesidades de éstos. De acuerdo con el estudio sobre "The state of journalism in 2011", realizado por Oriella PR Network (2011: web), los internautas opinan que las versiones digitales de los medios han superado a las versiones tradicionales en un 44 por ciento frente al 38 por ciento. Resultado que puede estar relacionado con el contenido que ofrecen las ediciones digitales que, según el estudio, en más del 50 por ciento de los casos se ha elaborado exclusivamente para la web.

Los periodistas no deben ni pueden quedarse al margen de las transformaciones que se están gestando con la Web social. Hoy, es ineludible su implicación en el proceso de información y comunicación que se está generando con los "social media", de ahí que el 31 por ciento de los periodistas españoles ya utiliza Twitter y el 28 por ciento Facebook para buscar y contrastar noticias, aunque admiten que no son muy fiables como lo revela el estudio realizado por Oriella PR Network. Estos porcentajes reafirman que el periodista es cada vez más consciente de la instauración de las redes sociales, del fortalecimiento de la sociedad civil y de su labor periodística dentro de esta encrucijada.

Las empresas de comunicación necesitan ser visibles en la Red a través de un proceso de negocio y no como una actividad aislada. Actualmente, la principal preocupación es establecer contacto con el usuario por medio de una comunicación relajada que permita instaurar una verdadera relación con el internauta para lograr la productividad. Y es que las redes sociales se han constituido como el reciente sistema operativo del comercio (Tapscott, 2008: 38-70). Está claro que las nuevas tendencias comunicativas están relacionadas con la actividad laboral de los distintos sectores profesionales y, por consiguiente, con las nuevas demandas de empleo.

Los profesionales de la comunicación deben asumir el reto que trae consigo la cultura digital y deben estar capacitados en el uso de nuevas herramientas tecnológicas que brindan las comunidades virtuales en la Red. También, implica la formación en competencias digitales y la adquisición de capacidades técnicas, sociales y cognitivas propias del entorno. "Las TIC no son sólo una red a la que se suman los individuos, sino que actúan como tecnologías sociales cuyo perfeccionamiento depende tanto de la diversidad de sus funciones (sociales, políticas, cognitivas, económicas, etc.) como de la flexibilidad con que se adapten a nuestra diversidad funcional" (Navarro, 2009: 141-148). Pero, ¿estos conocimientos se encuentran en las universidades españolas?

La presente investigación tiene como objetivo analizar el binomio formación universitaria y demanda de empleo por parte de las empresas para deslindar cuál es el requerimiento del mercado laboral en relación a los nuevos perfiles profesionales en comunicación que se han gestado en la Red. Por otro lado, es preciso conocer cuál es la propuesta educativa que ofrecen los másteres oficiales de las universidades españolas públicas y privadas. Seguidamente, determinaremos la correlación actual entre ambos o, en su defecto, el desencuentro y divergencia dentro de un mismo sistema o contexto social. 


\section{Material y métodos}

La presente investigación se ha desarrollado a lo largo de tres meses, de marzo a mayo de 2011, a la luz del alza creciente en la demanda de nuevos perfiles profesionales emergidos de la Web social durante el año 2010 y, especialmente, en los primeros meses del año 2011, según los datos obtenidos por los portales de empleo a los que hemos accedido, y ante la formalización de un nuevo mapa de másteres en las universidades españolas por la adaptación al Espacio Europeo de Educación Superior (EEES) durante el curso 2010/2011.

Dentro del marco de la demanda laboral en comunicación, que engloba el área de Publicidad, Marketing y Periodismo, delimitamos nuestro objetivo principal circunscribiéndolo al ámbito periodístico a la hora de contemplar la existencia de nuevos perfiles profesionales 2.0, de acuerdo a la correspondencia entre la formación universitaria especializada y la demanda laboral por parte de las empresas. En este sentido, las preguntas a las que pretendemos dar respuesta son: cuáles son los nuevos perfiles profesionales del periodista que se están gestando con la incursión de los medios sociales, qué nuevos perfiles profesionales demandan las empresas de comunicación y en qué medida existe una formación especializada y adaptada a las nuevas exigencias profesionales en las universidades españolas.

Planteamos como hipótesis de partida la presencia de los citados perfiles profesionales en la demanda laboral y la falta de correlación con la oferta educativa de las universidades españolas a través de másteres oficiales. Para conseguir nuestro objetivo, diseñamos una metodología basada en la utilización de técnicas cualitativas y cuantitativas en el estudio de un ítem, los nuevos perfiles profesionales 2.0, y en dos variables: la demanda laboral y la formación universitaria.

La evaluación sobre la demanda laboral de nuevos perfiles profesionales ha sido llevada cabo a través de técnicas cualitativas que nos han permitido adentrarnos en una fase de descubrimiento y están basadas en dos tipos de fuentes de información. Por una parte, recurrimos al análisis documental sobre la concepción y nominación de los nuevos perfiles profesionales y el contexto de emergencia en el que tienen lugar, así como estudios estadísticos aportados por el portal de empleo Infojobs, líder en su sector, que nos sirve de apoyo y guía de orientación sobre los nuevos perfiles profesionales 2.0.

Por otra parte, estos estudios aparecen complementados con las aportaciones de las fuentes primarias que representan dos miembros del personal de los portales de Infojobs y OficinaEmpleo, a los que se les ha presentado un formulario consistente en dos cuestiones: ¿cuáles son los nuevos perfiles en comunicación y en Periodismo, en particular, que más se están demandando actualmente? y ¿cuál es el número de demandas que recibió la figura del "Community Manager" y otras similares (Gestor de Contenidos, "Social Media Manager", "Social Manager Strategic", "Experto en Social Media", etc.) al cierre del año 2010?

Para investigar la oferta universitaria sobre los perfiles 2.0 nos servimos de técnicas cuantitativas que nos permiten justificar y confirmar la fase inductiva anterior. La muestra seleccionada nos concede un resultado cien por cien fiable, puesto que se 
trata del volumen total de los másteres oficiales de las 42 universidades españolas en las que se imparten estudios de Periodismo, de las cuales 19 son públicas y 23 son privadas, vigentes durante los cursos 2010/2011 y 2011/2012 y cuyos contenidos contemplan la formación de nuevos perfiles profesionales y/o giran en torno al marco de la Web social donde se desarrollan las herramientas que emplean. La recopilación de estos datos se ha realizado a través de las páginas web de las distintas universidades $\mathrm{y}$, en algunos casos, a través de la petición directa al personal responsable del máster.

El tipo de análisis estadístico empleado es el univariado cuando establecemos como variables los términos de presencia o ausencia en las universidades públicas y privadas de oferta de másteres que atañen a nuestro objeto de estudio y bivariado en el momento que relacionamos esta oferta con la comunidad autónoma en la que se encuentran las universidades y cuando conectamos la oferta universitaria en estudios de Periodismo y la oferta de másteres en formación de perfiles profesionales.

Con todo ello, conseguimos el principio de triangulación por el cual se emplean múltiples fuentes de evidencia que garantizan la validez interna de los resultados de acuerdo a la obtención de mayores dosis de fiabilidad, precisión, objetividad y rigor. Posteriormente, realizamos un estudio comparativo que nos lleva a formular una descripción certera sobre la situación real de la oferta universitaria en relación con la demanda de nuevos puestos de trabajo que exigen nuevos perfiles profesionales al periodista y que se extienden en las conclusiones.

\section{Perfiles profesionales bajo demanda}

Internet y las plataformas sociales han transformado las formas de recibir y transmitir la información gracias a las herramientas digitales y civiles que se desarrollan constantemente en estos espacios. Los medios sociales se han constituido como verdaderas plataformas ciudadanas donde es posible expresarse y debatir sobre diversas cuestiones de carácter económico, político, social, etc. Es más, se han constituido como un verdadero vehículo que canaliza la información y la movilización de las personas con conciencia democrática.

Asistimos en la Red a una revolución social y tecnológica que avanza a pasos agigantados. Los medios sociales se han arraigado en la vida de los ciudadanos y, a su vez, han ido ganando cuota de mercado. "Mientras que en 2007, 1 de cada 12 minutos en línea era dedicado a redes sociales, ahora 1 de cada seis minutos en Internet es invertido en los sitios de social media", dicta el informe realizado por ComScore', líder mundial en la medición de audiencia digital.

Es posible resumir las características que identifican la información que recorre por las comunidades virtuales que hoy son la columna vertebral de la Red: "La proximidad/hábitos, identificación temática, integración, familiaridad, servicio, participación, intercambio, reflexión sobre lo social, orientación, autonomía y convergencia-multimedia. Cada una de ellas tiene como fin producir una metacomu-

${ }^{1}$ Compañía de investigación de marketing en Internet fundada en agosto de 1999. Tiene como misión dar a conocer la conducta de los consumidores digitales, competidores y mercados para la estructuración de estrategias de venta. Véase en: http://www.comscore.com. 
nicación" (Sánchez y García, 2009: 381).

Debido al auge de la Web 2.0, más del 50 por ciento de empresas españolas han creído oportuno intensificar su inversión en nuevas herramientas tecnológicas propias de la Web Social hasta tal punto que el 68,6 por ciento de las compañías consideran que la financiación ha sido rentable. Así se desprende del informe "Las Tecnologías de la Información y las Comunicaciones en la empresa española 2010", efectuado por la Asociación de Empresas de Electrónica, Tecnologías de la Información y Telecomunicaciones de España (AETIC, 2010: web).

El proceso de despliegue de Internet y el impulso de los servicios digitales ha propiciado que se reactive el mercado de los medios de comunicación en España en 2010 y se prevea un crecimiento del 5,5 por ciento de media hasta 2014. El negocio atiende a los ingresos por acceso a Internet con un aumento del 14,8 por ciento, es decir, 12.017 millones de dólares hasta 2014 debido a la migración de los formatos digitales. Otro segmento en alza es la publicidad en Red con 10,5 por ciento hasta el 2014 y se posicionará en 1.521 millones de dólares. Así lo revela la undécima edición del "Global Entertainment and Media Outlook 2010-2014", elaborado por PwC (2010: web $)^{2}$. La empresa ofrece una percepción general de los medios a nivel mundial sobre su evolución en los próximos tres años.

No cabe duda de que las empresas impulsadas por el desarrollo de Internet tienen que innovar hacia nuevas fórmulas sostenibles. La innovación debe ser real y funcional para que les permita establecer sus estrategias de mercado en la Red. Independientemente del sector a la que pertenezcan, la pretensión se inclina hacia la presencia activa en los medios sociales, a pesar de que muchas compañías no tienen una idea clara de los fines que deben perseguir o las expectativas que aspiran alcanzar en estos espacios. La participación cada vez más activa de los ciudadanos en estas plataformas ha propiciado que las empresas innoven hacia nuevas tácticas empresariales. Ello ha impulsado la incursión de nuevos perfiles profesionales y concretamente en el ámbito de la comunicación. Empero, ¿cuáles son esos oficios bajo demanda?

En las plataformas digitales y sociales emergen distintas figuras profesionales. Actualmente, no existe uniformidad en determinar el nombre o el número de los perfiles que se han gestado. Veremos algunas clasificaciones realizadas por diferentes autores de libros y revistas, periodistas de cibermedios y blogs. La primera se corresponde con los siguientes perfiles profesionales, según Crucianelli (2010: 176-177): "Social Media Editor", "Community Manager", Reportero "Wifi”, Reportero Multimedia, "Data Base Finder", "Gestor AdSense", Administrador de Blogs, Ejecutivo "business" y Ejecutivo en Alianzas.

Entre las actividades realizadas con la Web 2.0, y más recientemente con la web en tiempo real, Jorge Pérez (2011: web) señala que se crean oportunidades de trabajo relacionados con: SEO "Specialist", "Community Manager", "Search Marketing Analyst", "Content Manager". En la misma línea, Castelló (2010, 74-97) cita los si-

2 Las industrias analizadas son: acceso a Internet (fijo y móvil), publicidad en Internet (fijo y móvil), televisión de pago, publicidad en televisión, prensa y videojuegos, entre otras. PwC ofrece servicios a 154 países a nivel mundial. 
guientes oficios: "Gestor de Social Media", "Community Manager", "Chief Blogging Officer", "Customer Insight" y "User Experience". Entre otros autores, cabe citar a Correyero y Baladrón (2010: web), quienes hacen hincapié en: SEO, "Community Manager" y "Social Media Manager", "News Developer", "Keyword Manager", "Search Editor" y "Newsroom Technology Manager".

Los perfiles profesionales 2.0 que aparecen en la Red no se caracterizan precisamente por ser multitarea o polivalente, afirma Marrero (2011: web), sino porque cambia "la organización de la producción ciberperiodística, la búsqueda de modelos de negocio, la centralidad de las concepciones y aplicaciones de la web 2.0, así como el empuje de la comunicación móvil”. Entre las labores periodísticas, el autor destaca: Editor de Medios Sociales, Gestor de Comunidades, Periodista Móvil, Reportero y Editor Multimedia, Administrador de Blogs, Editor de Datos, "Keyword Manager", entre otros.

También, podemos citar la clasificación realizada por periodistas de medios digitales o cibermedios, por ejemplo, Vázquez (2010: web) hace referencia a las siguientes demandas laborales: Director de comunicación 2.0, "Community Manager", Responsable de Reputación "Online", Experto en Analítica Web, Gestor de Contenidos Digitales, Arquitecto de la Información Digital y Experto en usabilidad.

No queremos dejar de lado la catalogación que se realiza desde los propios medios sociales, es decir, desde los blogs de los ciudadanos. Torzuko (2010: web) distingue los siguientes perfiles: Planificador de Medios Sociales ("Social Media Planner" u "On line Marketing Strategist"), Responsable de Reputación Digital ("Community Manager", "Online Reputation Manager" o "Chief Listening Officer"), Experto en SEO ("Search Engine Optimization"), Experto en SEM ("Search Engine Marketing") o PPC Manager, Responsable de Tráfico "Online" ("Traffic Manager" o "Trafficker"), Analista web ("Web Analytics"), Arquitecto de la Información Digital ("Information Architect"), Experto en Usabilidad Web, Gestor de Contenido ("Digital Content Manager o Content Curator") y Desarrollador o Diseñador Multimedia.

Otra bloguera, Dolores Vela, reconocida por su trayectoria profesional en "social media", destacó en el seminario "La Figura del "Community Manager" la Facultad de Comunicación de la Universidad de Sevilla los siguientes perfiles profesionales 2.0: "Social Media Strategies", "Social Media Manager"/"Strategist, Coolhunter" (caza tendencias), "Social Media SEO", "Content Manager" (edición y creación de contenidos) y "Content Curator" (selección de contenidos).

Dejando al margen la literatura sobre las distintas aportaciones de los teóricos, periodistas y blogueros, el estudio realizado por la Asociación Interactive Advertising Bureau y la empresa Cool Insights ${ }^{4}$ entre abril y mayo de 2011 da a conocer en la segunda oleada del "Observatorio del Mercado Laboral de los profesionales del marke-

3 El seminario fue emitido en directo en abril de 2011 en la Universidad de Sevilla bajo la dirección de la profesora Hada M. Sánchez Gonzales. Véase en: https://www.facebook.com /pages/Seminario-La-figura-del-Community-Manager/137742409628342?ref=ts

4 Es una compañía de investigación de consumidor y consultoría estratégica creada a mediados de 2009, Véase en: http://coolinsights.com/?page_id=79. 
ting, la comunicación y la publicidad digital" (IAB-Cool Insights, 2011: web) que, en el futuro, los perfiles profesionales relacionados con el apogeo de los medios sociales serán probablemente los más demandados.

El impacto de los medios sociales ha provocado oportunidades laborales relacionadas con los servicios y actividades sociales, propio de la Web 2.0 y más recientemente de la web en tiempo real. Impacto que va de la mano de la Tecnología de la Información y de la Comunicación porque permite crear diferentes modelos de negocio. Como se observa en la figura 1, se destaca la demanda de los Gestores de Comunidades o "Community Manager" con el 43 por ciento y, por otra parte, al responsable de Comunicación Digital/Marketing y Publicidad con el 19 por ciento. También, revela la necesidad de admitir profesionales responsables y expertos o estrategas especializados en medios sociales SMO, con el 14 y el 13 por ciento, respectivamente. Otros perfiles que podemos mencionar son: Expertos en SEO, SEM y Posicionamiento Web con el 12 por ciento; Desarrolladores Web y de Aplicaciones con el 11 por ciento y Expertos en Analítica Web con el 7 por ciento.

Según los datos aportados por Eduardo Ruiz, representante del portal OficinaEmpleo, "los perfiles más demandados por las empresas son los de aquellas personas que tienen conocimientos medios-altos en las nuevas herramientas de comunicación que ofrece Internet. Concretamente, el puesto de "Community Manager", es uno de los perfiles más solicitados. También llamados "dinamizadores sociales", son los encargados de trasladar a las redes sociales las estrategias de marketing y comunicaciones de una empresa. También se demandan "blogueros" y redactores de contenidos para los blogs de las empresas". Y añade que la demanda del perfil de "Community Manager" y de gestor de contenidos sobrepasó en 2010 las 1.000 visitas.

Sin embargo, ¿qué datos revelan los portales de empleo al que acuden los ciudadanos a la hora de buscar un puesto de trabajo? Según el Panel de Hogar y Trabajo, realizado por Nielsen Online España correspondiente al mes de abril de 2011, Infojobs lidera el ranking de audiencia con 2,1 millones de usuarios únicos de un total de 4,8 millones de internautas que consultaron los portales de trabajo, un 6 por ciento menos que en el mismo período del año anterior ${ }^{5}$. Es posible que se deba a que los internautas apuestan por nuevas fórmulas para buscar empleo. Las redes sociales profesionales como Linkedin o Xing se han constituido en una alternativa que aporta valor en la búsqueda de trabajo aunque en un inicio su uso se limitaba a la selección de perfiles cualificados a diferencia de hoy, cuando más del 50 por ciento de españoles y el 80 por ciento de directivos de recursos humanos utilizan las redes sociales para buscar empleo y candidatos, según el estudio ${ }^{6}$ de Viadeo, Red Social para profesionales.

Infojobs, con 2,1 millones de usuarios únicos, se encuentra con diferencia por encima de los demás portales de empleo (Infoempleo, Trabajo.com, Jobrapido, Minis-

5 Lara Recuero, consultora estratégica especializada en comunicación, gabinete de prensa y lobby de la compañía "Ion, Imagen y Comunicación" argumenta que posiblemente se deba a la coincidencia con los días festivos de Semana Santa.

${ }^{6}$ Estudio en el que han participado más de 2.000 españoles y 50 directivos de recursos humanos. 
terio de Trabajo e Inmigración y Laboris.net) más utilizados por los internautas (Ion, Imagen y Comunicación, 2011: web). Por ello, nuestra investigación se centra en la bolsa de oficio líder de España para dar respuesta a la interrogante formulada en el párrafo anterior, ¿qué datos revelan los portales de empleo sobre la demanda de los nuevos perfiles profesionales? El estudio titulado las "Profesiones relacionadas con Internet en el mercado laboral español 2010", llevado a cabo por Infojobs y la Asociación Española de la Economía Digital y publicado en febrero de 2011 (InfojobsAdigital, 2011: web), desvela que los expertos que trabajan en el soporte de Internet están mejor remunerados que el resto de profesionales de su categoría. Esta diferencia es aún más significativa en el sector del Marketing y Comunicación donde se paga un 21 por ciento más.

Asimismo, el estudio señala que son cinco las categorías profesionales que concentran la demanda de nuevos profesionales de Internet: Informática y Telecomunicaciones, Diseño y Artes gráficas, Comercial y Ventas, Atención al Cliente y Marketing y Comunicación. Este último está relacionado con "las redes sociales, los programas de afiliación, las comunidades online, el diseño y la forma de interactuar y la búsqueda de negocio a través de la web". Algunos de las perfiles profesionales agrupados dentro de esta categoría son: "Community Manager", Experto en "Social Media", Especialista SEO, Especialista SEM, "Traffic Manager", "Web Business Developer", Analista de Tráfico ("Traffic Analyst”), etc. Además, Infojobs (2011: web) indica que el perfil de "Community Manager" está en alza porque el número de vacantes en 2010 (164 puestos de trabajo) se multiplicó por ocho con respecto a las ofertadas en 2009 ( 21 puestos de trabajo).

Visto el panorama, según la revisión teórica efectuada y los estudios publicados por Interactive Advertising Bureau (IAB)-Cool Insights, Infojobs y la Asociación Española de la Economía Digital (Adigital), es posible resumir que los perfiles con mayor demanda laboral son "Community Manager" y "Social Media Editor" por encima del resto, como también lo señalan autores como Tuñez, Martínez y Abejón (2010: 79-94), Flores (2009: 73-81) y la Asociación Española de Responsable de Comunidad ${ }^{7}$ (AERCO). Por otro lado, se ha podido observar que no existe consenso en la denominación de los nuevos perfiles 2.0, pues una misma función es posible que tenga dos o tres designaciones como ocurre, por ejemplo, con el perfil de "Digital Content Manager" identificado también como "Content Curator", etc. También, es evidente el amplio abanico de nuevas profesiones que se han gestado en Internet debido a la evolución de la Web 2.0 y de los medios sociales.

Igualmente, es posible corroborar estos datos con las propias herramientas sociales. Es el caso del buscador más utilizado por los internautas, Google. Es admisible comprobar a través de Google Insights el volumen de búsquedas que recibe una determinada palabra clave como "Community Manager" en la Red. España es el primer país del mundo en requerir información sobre este nuevo perfil profesional, seguido

7 AERCO nace en 2008. Es una "entidad sin ánimo de lucro compuesta por profesionales relacionados con las comunidades virtuales y que tiene la misión de atender las necesidades de los responsables de comunidades online". Véase en: http://www.aercomunidad.org 
de Estados Unidos y Australia (Google Insights, 2011: web). Las provincias españolas que más solicitan este tipo de profesional, según Infojobs (2011: web), son Madrid con el 53 por ciento del total de ofertas publicadas y Barcelona con el 33,5 por ciento.

Si comparamos la evolución de los porcentajes registrados desde el 2004 hasta junio de 2011 en relación con el número de búsquedas realizadas por el internauta sobre el perfil profesional "Community Manager", es perceptible que en España el auge del reciente oficio se ha producido a partir del 2010 (Google Insights, 2011: web). Como ya se ha mencionado, la demanda de la oferta de empleo se multiplicó por ocho en 2010 con respecto al 2009 y en 2011 adquiere mayor reclamo por parte de los propios ciudadanos. Sin embargo, no ocurre lo mismo en otros países como Estados Unidos o con el resto de europeos, donde incluso no sólo es posible exponer la figura del "Community Manager" como un perfil laboral, sino como una función más dentro de un puesto concreto.

Por su parte, Eduardo Ruiz, representante del portal OficinaEmpleo, reafirma que el "boom" del perfil de "Community Manager" fue el año 2010 "pero es este 2011 en el que se está consagrando como un puesto de relevancia tanto en empresas como en agencias de publicidad. En lo que llevamos de año, las visitas a OficinaEmpleo en busca de ofertas para este perfil son las mismas que en todo el año pasado. Por lo que preveemos que al finalizar el año, dicho perfil laboral habrá tenido más del doble de la presencia que tuvo en 2010".

Pero, ¿qué formación se exige para estos puestos laborales? Lo más requerido son los diplomados o licenciados con un 48,2 por ciento del total de las ofertas, seguido de estudios con máster con un 15,2 por ciento, mientras un 15 por ciento de las vacantes ofertadas no exigen estudios específicos. Las licenciaturas más valoradas son Periodismo, Marketing y Publicidad. En relación a los requerimientos para cubrir estas vacantes, el 27 por ciento de las ofertas no precisan experiencia alguna, el 24,78 por ciento de los puestos ofertados exige al menos dos años de experiencia en el sector y el 24,74 por ciento solicitan un mínimo de un año, como lo recoge el informe realizado por Infojobs y la Asociación Española de la Economía Digital (2011: web).

Es evidente que la evolución de los medios sociales trae consigo fuertes repercusiones en la demanda del mercado laboral y de acuerdo a los conocimientos que debe adquirir el profesional que desee optar a una vacante. El estudio de Interactive Advertising Bureau (IAB)-Cool Insights (2011: web) evidencia en este sentido la necesidad de una formación concreta por parte de los profesionales en torno a: medios sociales, Web 2.0 y "cloud computing" sobre el resto de áreas que ostentan el 40 por ciento; SEO/SEM/buscadores con el 13 por ciento; programación/desarrollo Web, 7 por ciento; analítica/métricas, 7 por ciento o "mobile advertising" con el 6 por ciento, etc. A pesar de ello, es posible que la tendencia esté en consonancia con la mejora de la adquisición de conocimientos aprendidos por el interés que demanda la Web social y por el promedio del sueldo ofertado, un 21 por ciento más que en el resto de ofertas de esta categoría, según el informe de Infojobs y la Asociación Española de la Economía Digital (2011: web). A partir de este momento cabe preguntarse por la oferta educativa que ofrecen los másteres oficiales de las universidades españolas y si los nuevos perfiles profesionales cuentan con una formación reglada. 


\section{Discusión}

Pese a las diversas denominaciones de puestos de trabajo emergidos en el contexto de la Web social, la figura del "Community Manager" se erige como la más utilizada por el mercado laboral y por la oferta universitaria para hacer alusión a un mismo perfil, seguido del "Social Media Editor". Así lo reafirma el auge iniciado en el año 2010 y que se consagra en los años 2011 y 2012.

Este hecho, junto al creciente porcentaje de demandas laborales de diplomados o licenciados con máster, cifrado en un 15,2 por ciento, implican que la oferta de másteres especializados en nuevos perfiles profesionales en las universidades garantizan en alto porcentaje que un recién titulado en Periodismo pueda acceder al mercado de trabajo, más aún, si atendemos a que en un 48,2 por ciento la demanda para cubrir puestos con este tipo de perfiles es de diplomados o licenciados en Periodismo, Marketing y Publicidad y a que en un 27 por ciento no se precisa experiencia previa alguna, el 24,78 por ciento de los puestos exige al menos dos años de experiencia en el sector y el 24,74 por ciento un mínimo de un año.

Las universidades que presentan contenidos especializados en perfiles 2.0, especialmente sobre la figura del "Community Manager", son de titularidad privada y están concentradas en tres comunidades autónomas (Madrid, Cataluña y Navarra), lo que indica que las universidades privadas han comenzado a presentar en mayor proporción una oferta sobre comunicación multimedia con la que los futuros periodistas podrán competir en mejores condiciones en el mercado, mientras que en las universidades públicas se contempla un desfase en la adaptación a las nuevas necesidades laborales de los futuros profesionales del Periodismo que se desarrolla en la Red. En términos generales, se precisa un ajuste entre la oferta universitaria especializada en nuevos perfiles de trabajo de acuerdo a la demanda de las empresas para conseguir un desarrollo equiparado de la actividad profesional periodística.

\section{Referencias bibliográficas}

AETIC (2010): "Las Tecnologías de la Información y las Comunicaciones en la empresa española 2010": http://www.aetic.es/es/inicio/actualidad/58/contenido.aspx [fecha de consulta: 30 de abril de 2011]

AIMC (2011): "Estudio General de Medios 1 ${ }^{a}$ Ola, febrero/marzo": http://www.aimc .es//-Datos-EGM-Resumen-General-.html [fecha de consulta: 15 de abril de 2011].

CASTELLÓ, Araceli (2010): "Una nueva figura profesional: el Community Manager", en Pangea, $\mathrm{n}^{\circ} 1$, pp. 74-97: http://revistaraic.files.wordpress.com/2010/12/0101-104.pdf. [fecha de consulta: 21 de abril de 2011]

CORREYERO, Beatriz y BALADRÓN, Antonio (2010): "Nuevos perfiles profesionales en el entorno digital: un desafío para la formación de comunicadores desde el EEES", en AA. VV: Actas II Congreso Internacional Latina de Comunicación Social: http://www.revistalatinacs.org/10SLCS/actas_2010/044_Correyero.pdf. [fecha de consulta: 15 de abril de 2011]

CRUCIANELLI, Sandra (2010): Herramientas digitales para periodistas. Texas, University of Texas at Austin, Knight Center for Journalism in the Americas. 
GOOGLE INSIGHTS (2011): "Interés de búsqueda en la Web: community manager": http://www.google.com/insights/search/\#q=COMMUNITY\%20MANAGER\%2C\&cmpt=q [fecha de consulta: 14 de abril de 2011]

IAB-COOL INSIGHTS (2011): "Observatorio del Mercado Laboral de los profesionales del marketing, la comunicación y la publicidad digital", $2^{\mathrm{a}}$ oleada, abril y mayo de 2011: http://www.iabspain.net/ver-php?mod=noticias\&identificador=100 [20 de abril de 2011]

IAB-ELOGIA IPSOFACTO (2010): "II Estudio sobre Redes Sociales en Internet": http://www.iabspain.net/ver.php?mod=noticias\&identificador $=80$ [fecha de consulta: 16 de mayo de 2011]

INFOJOBS-ADIGITAL (2011): "Profesiones relacionadas con Internet en el mercado laboral español 2010": http://www.adigital.org/resources/image/ProfesionesInternet_MdoLaboral2010.pdf. [fecha de consulta: 20 de abril de 2011].

INSTITUTO NACIONAL DE EMPLEO (2010): "Equipamiento y Uso de Tecnologías de Información y Comunicación en los Hogares": http://www.ine.es /jaxi/menu.do?type $=$ pcaxis\&path $=/ \mathrm{t} 25 / \mathrm{p} 450 \&$ file $=$ inebase $[$ fecha de consulta: 14 de abril de 2011]

ION, IMAGEN Y COMUNICACIÓN (2011): "Ranking de portales más utilizados por los internautas": http://www.ioncomunicacion.es [fecha de consulta: 17 de abril de 2011]

FLORES, Jesús Miguel (2009): "Nuevos modelos de comunicación, perfiles y tendencias en las redes sociales". Comunicar, $\mathrm{n}^{\circ} 33$. Huelva, Grupo Comunicar, Colectivo Andaluz de Educación en Medios de Comunicación, pp. 73-81.

MARRERO, Liliam (2001): "Gestor de comunidades, reportero móvil, desarrollador..., o sea, periodista", en La Jiribilla, $\mathrm{n}^{\circ}$ 506: http://www.lajiribilla.co.cu /2011/n506_01/506_02.html [fecha de consulta: 26 de mayo de 2011].

NAVARRO, María, G. (2009): "Los nuevos entornos educativos: desafíos cognitivos para una inteligencia colectiva". Comunicar, $\mathrm{n}^{\circ} 33$. Huelva, Grupo Comunicar, Colectivo Andaluz de Educación en Medios de Comunicación, pp.141-148.

ORIELLA PR NETWORK (2011): "The state of journalism in 2011": http://www.oriellaprnetwork.com [fecha de consulta: 18 de mayo de 2011]

PÉREZ, Jorge (2011): "Nuevos yacimientos de empleo y formación”, en Telos, no 87: $\mathrm{http}: / /$ sociedadinformacion.fundacion.telefonica.com/seccion $=1266 \&$ idioma $=$ es_E S\&id=2011051112430001\&activo $=6$.do [fecha de consulta: 24 de mayo de 2011]

PwC (2010): "Global Entertainment and Media Outlook 2010-2014": http://www.pwc.com/es/es/publicaciones/informes-entretenimiento-medios.jhtml [fecha de consulta: 20 de mayo de 2011]

SÁNCHEZ, Hada M. y GARCÍA, Noelia (2009): "Las redes sociales, la información emotiva y la participación especializada", en QUESADA PÉREZ, Montserrrat (ed.): Internet como fuente generadora de contenidos especializados. Barcelona, Universidad Pompeu Fabra, Sociedad Latina de Comunicación Social e Instituto de Estudios de Comunicación Especializada, pp. 371-387. 
TAPSCOTT, Don (2008): Grown Up Digital: How the Net Generation is changing your world. New York, McGraw-Hill.

TORZUKO (2010): "Nuevos perfiles profesionales 2.0", en Torzu 3.0: http:/torzuko.wordpress.com/2010/08/18/nuevos-perfiles-profesionales-2-0 [fecha de consulta: 16 de mayo de 2011]

TÚÑEZ, Miguel; MARTÍNEZ, Yolanda y ABEJÓN, Paloma (2010): "Nuevos entornos, nuevas demandas, nuevos periodistas". Estudios sobre el Mensaje Periodístico, $\mathrm{n}^{\mathrm{o}}$ 16. Madrid, Servicio de Publicaciones de la Editorial Complutense, pp. 79-94.

VÁZQUEZ, Tamara (2010): "Perfil y salario medio de los profesionales 2.0", en Expansiónyempleo.com: http://archivo.expansionyempleo.com/2010/06/11/desarro1lo_de_carrera/1276279664.hml [fecha de consulta: 12 de mayo de 2011]

\section{Hada M. SÁNCHEZ GONZALES}

Universidad de Sevilla

Facultad de Comunicación. Departamento Periodismo II.

Profesora Contratada Doctora

misago@us.es

\section{Sandra MÉNDEZ MUROS}

Universidad de Sevilla

Facultad de Comunicación. Departamento Periodismo II.

Profesora Sustituta Interina

sanmenmur@us.es 